\title{
LA DIFERENCIACIÓN POR CALIDAD: ¿CUÁLES SON LOS LÍMITES?
}

Alejandro Sotomayor* Alfonso Muga* 


\section{RESUMEN}

En este artículo se reflexiona en torno al fenómeno de la diferenciación por calidad. En primer lugar, se distingue entre la calidad de la carrera, como unidad organizacional, y la calidad del egresado al ingresar al mercado laboral. La incorporación de profesionales y técnicos con competencias $\mathrm{y}$ conocimientos inferiores a las atendibles en un esquema de educación de elite, es uno de los aspectos más controversiales de la masificación de la educación superior, si bien hay un espacio posible para la diferenciación. Todo este proceso puede ser beneficioso, pero es conveniente establecer ciertos límites dado que hay buenas razones para ello. Finalmente, se formulan propuestas para el mejoramiento de los sistemas de aseguramiento de calidad, las que se desprenden de la distinción entre calidad de la carrera y calidad del egresado.

\section{ABSTRACT}

This paper examines the subject of differentiation through quality. First, it distinguishes between the quality of the study program, as an organizational unit, and the quality of graduates when they enter the labor market. The incorporation of professionals and technicians equipped with below-par competencies and knowledge to those worthy of consideration in an elite education scheme is one of the most controversial aspects in the massification of higher education, although this provides space for differentiation. The entire process can prove beneficial but certain limits need to be established because there are good reasons to do so. Finally, the paper proposes systems to improve quality assurance stemming from a distinction between the quality of the study program and the quality of the graduate. 


\section{LA DIFERENCIACIÓN POR CALIDAD: ¿CUÁLES SON LOS LÍMITES?}

Calidad de la carrera y calidad del egresado: una precisión

El sistema chileno de educación superior ha experimentado en las últimas décadas un proceso de masificación y diferenciación (Muga y Brunner, 1996; Bernasconi y Rojas, 2004). Una de las consecuencias de este proceso es la heterogeneidad en la calidad de la oferta de carreras de todo nivel, lo que conduce a diferencias relevantes entre instituciones que formalmente reciben igual denominación, tal como ocurre en Estados Unidos y Japón (Teichler, 1996).

\section{Conceptos de calidad}

La calidad de una carrera, en cuanto oferta institucional, no debiera ser confundida con la calidad del egresado que ha sido formado en ella. Como se puede apreciar en Lomas (2001), Harvey (1999), y Lagrosen, Seyyed-Hashemi y Leitner (2004), hay diversas formas de interpretar el concepto de calidad.

La calidad del egresado está en directa relación con la valoración que de él hace el mercado laboral, la cual es, en parte, atribuible al título profesional o técnico que porta, puesto que hay evidencias de que no toda la valoración depende de tal atributo: otras características personales, así como diversas circunstancias externas tanto al egresado como a la institución -prejuicio, por ejemplo-, influyen en diferente medida (Núñez y Gutiérrez 2004).

La calidad de la carrera está en directa relación con la promesa que hace la institución a sus alumnos, como principales beneficiarios 
de la formación recibida. El primer test de calidad que la institución debe pasar es el cumplimiento de dicha promesa. Esta puede ser medida en términos de valor agregado: el joven ingresa con una determinada posición comparativa (por ejemplo, su ubicación en los resultados de la prueba de selección universitaria) y egresa con otra (medida por la valoración que de él hace el mercado laboral atribuible a su formación). La ganancia o pérdida de posiciones es el valor agregado (o disminuido) que puede ofrecer la institución. Un enfoque de valor agregado permite medir tanto la efectividad de las instituciones como su eficiencia, comparando el valor agregado creado por la institución con el costo incurrido en obtenerlo (aranceles, tiempo, esfuerzo, descarte de alternativas accesibles). En definitiva, la calidad de la institución puede ser entendida, desde esta perspectiva, como la capacidad de la entidad para entregar a un alumno el valor agregado prometido al costo acordado.

Puede haber distintas combinaciones de calidad de carrera y calidad del egresado, como se muestra en el diagrama siguiente:

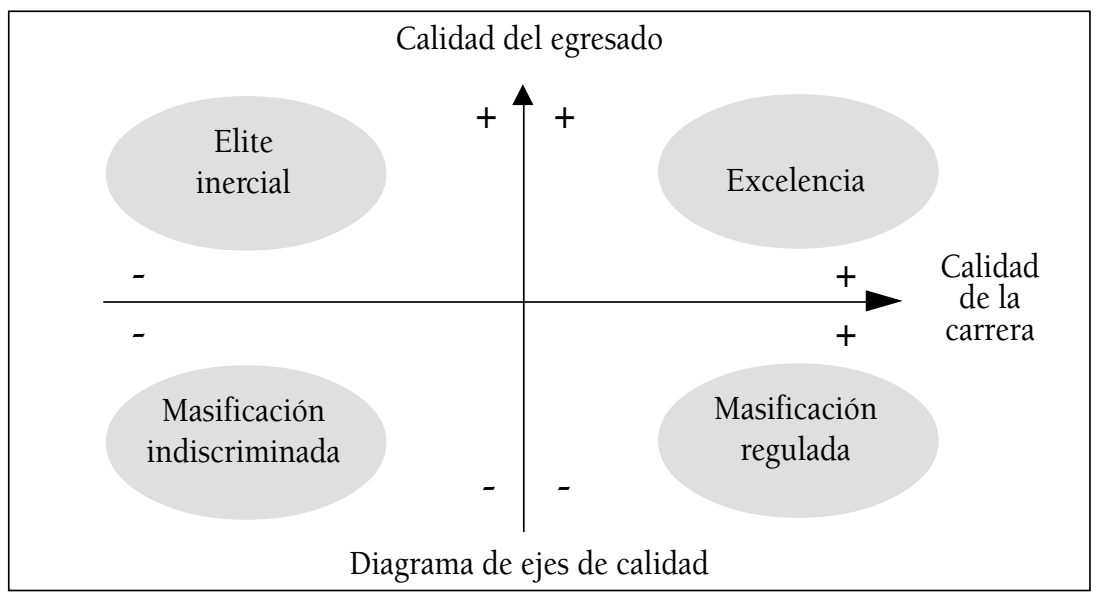

La diferenciación por calidad puede tomar dos direcciones: una, coexistencia de carreras que asumen responsablemente las promesas que hacen a los estudiantes junto con carreras que no cumplen sus promesas o no monitorean dicho cumplimiento, y dos, coexistencia de carreras que forman profesionales y técnicos de un nivel de excelencia junto con carreras que forman profesionales y técnicos de 
un nivel más masivo (los que, eventualmente y a igualdad de otros factores, accederían a puestos de trabajo con menores responsabilidades e ingresos).

La diferenciación por calidad suele ser vista como una distorsión. Distinguir, entonces, entre dos formas de diferenciación por calidad ayuda a focalizar mejor las preocupaciones. Por una parte, es conveniente establecer estándares de calidad institucional que todos debieran cumplir si se aspira a un mercado de educación superior idealmente libre del fraude. Por otra parte, hay que reflexionar sobre cuáles son los límites para la diferenciación por calidad del egresado, reconociendo previamente que este tipo de diferenciación presenta algunos beneficios tanto para los jóvenes como para los empleadores.

Espacio para la diferenciación

Hay razones para pensar que, tanto para los postulantes a la educación superior como para los empleadores, resulta beneficiosa una diferenciación por calidad del egresado. Por tratarse ésta de una estratificación que se produce al interior de un determinado nivel institucional (por ejemplo, universidades) se la denominará "diferenciación diagonal".

La perspectiva de los estudiantes

La masificación de la educación superior obliga a las instituciones a adaptar sus programas para alumnos con diferentes habilidades y expectativas (Randal, 2002). Desde la perspectiva de los postulantes a la educación superior, las razones para valorar la existencia de distintos niveles pueden ser, entre otras, las diferentes expectativas de los jóvenes respecto a sus vidas, las distintas posibilidades de emprender proyectos académicos y los variados recursos, externos al programa institucional, con que complementarán su preparación en vistas a los requerimientos del mercado laboral.

Diferencia de expectativas. Algunos jóvenes, movidos por una fuerte vocación, están dispuestos a dedicar su juventud al cultivo de una carrera; otros esperan obtener medios para un fin (dinero, poder, prestigio, por ejemplo); y otros buscan la seguridad económica en sus 
vidas, que proyectan como no necesariamente vinculada al trabajo y al estudio. ¿Hay una perspectiva moralmente superior a las otras en esta materia? Muy probablemente no, como tampoco parece haber razones para obligar a los jóvenes a asumir un nivel profesional homogéneo: a algunos se les exigirá más de la cuenta-o derechamente se les excluirá-, a otros se les impedirá satisfacer su ambición intelectual.

Diferencia de posibilidades. En primer lugar, hay diferencias en las posibilidades económicas, lo que sólo puede ser parcialmente subsanado en el sistema público. Agréguese a ello que no todos están dotados con la misma inteligencia, por lo que se necesita graduar las metas y disponer de sistemas acordes a los estilos de aprendizaje (más autónomo o más asistido, más abstracto o más experimental, por ejemplo).

Diferencia de recursos complementarios. Los jóvenes cuentan con diferentes recursos complementarios que, junto a la formación profesional que reciban, constituirán su oferta al mercado laboral. Saben que con el título no se juegan su futuro, sino sólo parte de él. Tienen ya en la mano, o se encuentran cultivando (a veces en el tiempo que deciden no dedicarle a la educación formal) otras competencias o conocimientos, como idiomas, tecnología, artes o deportes: campos del saber que no calzan con las carreras existentes. Han acumulado (o están acumulando) experiencia, por trabajar desde pequeños, con su familia o en otros ambientes, en un rubro determinado. Tienen una personalidad atractiva para ciertos empleos o poseen "inteligencia emocional". O, lisa y llanamente, cuentan con recursos externos a sus decisiones y a la formación académica recibida, ya sea patrimonio (actual o herencia potencial) o adecuadas relaciones sociales para tener éxito si se cuenta con un estándar determinado de formación.

La perspectiva de los empleadores

Así como los jóvenes, las empresas tienen buenas razones para valorar un sistema en el que haya diferenciación diagonal. La primera es que las necesidades de profesionales son heterogéneas: no siempre necesitan un profesional de punta para resolverlas. Bastantes puestos, por ejemplo, son rutinarios o altamente especializados. Contratar para ellos profesionales de un segmento altamente valorado por el mercado 
sería incurrir en una ineficiencia tanto privada como social. Hay empleos (como trabajos otrora "de carrera") que se han profesionalizado recientemente, lo cual resulta ser natural cuando aumenta la cobertura universitaria. Hay, asimismo, ubicaciones laborales fuertemente vinculadas a las relaciones interpersonales (como las áreas de gestión y ventas), en las que se necesita un profesional con atributos obtenidos en la educación superior, de carácter básico y transversal. Para ellos se requiere a alguien que tenga métodos de trabajo, capacidad de aprendizaje y de resolución de problemas, así como cierto nivel de cultura general y conocimiento de su entorno y del de la empresa. Alguien que haya logrado un nivel importante de competencia en aspectos como desempeño lingüístico (en la lengua propia y en alguna extranjera) y computación.

En la medida en que las carreras ofrezcan una formación general y una especializada, habrá empleadores que se darán por satisfechos al contratar a quienes hayan recibido una buena formación general, prescindiendo del resto y asegurando una distribución eficiente de los recursos humanos.

\section{Límites a la diferenciación}

Hasta aquí se han dado razones para considerar valioso un cierto margen de diferenciación diagonal. ¿Es ilimitado este margen? De existir: ¿dónde debiera situarse tal límite? ¿A quién o a quiénes le corresponde fijarlo, si es el caso?

\section{Razones para fijar límites}

Si bien hay quien piensa que los límites sólo debieran ser fijados por el mercado, ya que éste se encargaría de eliminar los proyectos que no generen un valor superior al costo que exigen, hay al menos una restricción en la que prácticamente todos estarán de acuerdo: las instituciones deben formular con claridad sus promesas a los usuarios y éstas deben ser satisfechas. Asimismo, para analizar la valía de las distintas ofertas, deben reducirse las asimetrías de información y permitir comparaciones. Nadie se beneficia de un sistema en el que la publicidad engañosa sea válida y en el que los jóvenes deban tomar a ciegas decisiones trascendentales; nadie, salvo especuladores cuyos intereses no tiene sentido defender. 
Aparte de esta exigencia de transparencia -cuyo principal efecto es la necesidad de generar y hacer disponible volúmenes cada vez más grandes de información-, hay otras razones por las cuales es preciso que se adopten restricciones, más allá de la voluntad del mercado. Una de ellas es que en la educación superior está comprometida la fe pública en los títulos. A menudo se dice que el mismo mercado excluirá a aquellos portadores de títulos sin valor, pero esto supone una capacidad que, eventualmente, sólo tienen las grandes empresas. Hay portadores de títulos cuyas negligencias pueden significar la muerte de personas: médicos, ingenieros civiles, técnicos en instalaciones de gas y electricidad, por ejemplo. En otros casos los usuarios se juegan su propiedad o incluso su libertad, como ocurre con el desempeño de abogados.

Es necesario, entonces, un estándar mínimo de conocimientos y competencias que debe ser satisfecho por todos los portadores de un mismo título, al menos en aquellos títulos cuyo desempeño tiene consecuencias importantes para sus posteriores usuarios. Un parámetro razonable para fijar estos estándares puede ser situarlos en el segmento superior del nivel vertical inmediatamente inferior; por ejemplo, un ingeniero civil de la gama inferior debe ser a lo menos comparable a un ingeniero de ejecución de la gama superior y, a la vez, un ingeniero de ejecución de la gama inferior debe ser a lo menos comparable con un técnico de la misma especialidad. La estrictez con que se adopten estos estándares mínimos, de carrera en carrera, es una característica propia de la definición a realizar. Así, para algunas carreras como, por ejemplo, la de médico, debiera haber poco margen de diferenciación diagonal.

Otra razón para introducir restricciones es la necesidad de velar por el buen uso de los recursos públicos cuando hay subsidios comprometidos. Estos subsidios se otorgan porque detrás de la transacción respaldada hay un beneficio para la comunidad (disponibilidad de un profesional o técnico) y/o porque hay un acto de justicia para con el subsidiado (acceso a la educación superior para poder insertarse en el mercado laboral). Si no se produce ni una cosa ni la otra, la oferta de que se trate no debiera al menos contar con posibilidades de financiamiento público. 
Una razón adicional para establecer límites a la diferenciación diagonal es la conveniencia de establecer incentivos y presiones que eleven sistemáticamente la competitividad del país a partir de la competitividad del capital humano. Tanto por esta última razón como también por todas las anteriores, las restricciones establecidas tienen un carácter dinámico y debieran ser modificables con el paso del tiempo.

Determinación de límites y actores involucrados

La pregunta a abordar es qué rol y qué capacidad tiene cada involucrado en la fijación de límites.

Del Estado se esperan varias tareas: velar por la fe pública, cautelar el buen uso de los recursos fiscales, asegurar la corrección de las transacciones del mercado (impedir o castigar el fraude, principalmente) y promover la competitividad del país. Estas expectativas se vuelven difíciles de cumplir en la medida en que las materias reguladas se hacen más complejas de supervisar, como la calidad de la educación o la pertinencia de la oferta.

Del mercado se espera que discrimine las ofertas buenas de las malas, para lo cual requiere información que le permita evaluar y comparar. Además, requiere una importante capacidad de análisis e información previa, la cual, al menos desde la demanda de jóvenes y padres de familia, implica un proceso de aprendizaje que suele ser lento y asimétrico.

De las instituciones, se espera que se desenvuelvan con pericia en la planificación y supervisión de sus actividades, y con un decidido nivel ético en el cuidado de su gestión (responsabilidad social). Probablemente sea en las instituciones donde más radica la capacidad de medir y controlar la calidad; aunque esta capacidad, no obstante, puede verse opacada por conflictos de intereses o por juegos internos de poder.

Las agencias de acreditación tienen el desafío no sólo de evaluar la calidad, sino de percibir qué aspectos deben ser sopesados en función de las necesidades de la sociedad, a la par con saber transmitir los resultados de sus procesos de una manera simple y con alto impacto. 
De los medios de comunicación se espera información clara, veraz y pertinente. Ellos tienen la posibilidad de llegar a la mayor parte de las familias y de recabar información por parte de las instituciones. Este rol puede verse afectado por restricciones de capacidad, dadas por la falta de especialización en la materia de los periodistas, o por conflictos de intereses con la inversión publicitaria.

De la sociedad en su conjunto se espera un mayor interés por el tema. La educación superior no es sólo un asunto que atañe a los padres de jóvenes en edad de acceder a ella; involucra a todos, por cuanto determina tanto la calidad de los profesionales y técnicos requeridos, como también la competitividad del país y, por lo tanto, las expectativas de calidad de vida.

\section{Propuestas}

Asegurar calidad en la educación superior requiere la consolidación de confianzas entre los distintos actores. Más que un "sistema" de aseguramiento de calidad, lo que se necesita es una "cultura" de aseguramiento de calidad en la que participen todos los involucrados. En pos de la construcción de esta cultura, es posible formular las siguientes propuestas:

- Profundizar los estudios que permitan conocer bien la heterogeneidad de las instituciones, en cuanto a la calidad de sus egresados. Como sugieren Raczynski y Canales (2000), las perspectivas de empleabilidad e ingreso asociadas a una carrera específica, impartida por una determinada institución, necesitan ser conocidas por los postulantes. No es una tarea fácil ni rápida: se requiere información así como modelos analíticos. El trabajo desarrollado hasta la fecha por estudiosos como Meller y Brunner ${ }^{1}$ es un buen ejemplo de lo que se puede obtener (aunque los resultados que se entregan, más que como un producto final, debieran ser vistos como los propios de un proyecto que requiere ser escalado).

- Adoptar como regla de transparencia que hay un nivel apropiado de información que no coincide necesariamente con el máximo. Hay un punto en que el aumento de información

1 Ver el sitio en internet www.futurolaboral.cl 
se convierte en entropía, pues deja de ser utilizable. Por lo tanto, entregar volúmenes gigantescos de información y "dejar que el mercado funcione" no es hacerlo más transparente. En los espacios amplios de debate antes mencionados, es necesario concordar ciertas fórmulas que contribuyan a simplificar la información que se entrega y hacerla realmente valiosa para los jóvenes y sus familias.

- Adaptar tanto los mecanismos de entrega de información como los procesos de acreditación a la constatación de que la calidad de la formación no es igual a la calidad del egresado. En los procesos de acreditación de carreras se está acumulando una experiencia que no está necesariamente considerando estas características diferenciadoras.

- Construir un gran pacto de confianza entre la sociedad y las instituciones educacionales. Considerando la alta complejidad del aseguramiento de la calidad, es muy difícil que ésta pueda ser hecha en forma externa a las instituciones; pero tampoco es razonable esperar que la calidad sea asegurada por la única voluntad de ellas. Las instituciones deben recibir autonomía y entregar accountability. El Estado, las agencias de acreditación y los medios de comunicación son buenos mediadores entre la sociedad y las instituciones, buenos "testigos" de la seriedad con que se realiza el trabajo. La constitución de sistemas interinstitucionales permite despejar las dificultades que tienen las entidades para autocontrolarse o autorregularse.

- Ordenar la estructura institucional, hoy diseñada para la existencia de tres niveles (universidades, institutos profesionales y centros de formación técnica) que, traslapadamente, ofrecen dos niveles de títulos (profesionales y técnicos). Por otra parte, la formación de elite puede ser orientada hacia niveles superiores (posgrado y formación continua), reconociendo que la aspiración de la presente década es más la educación masiva que la educación de elite de tres décadas atrás.

- Por último, en la medida en que se midan indicadores relevantes respecto de la calidad de la carrera ofrecida, es 
conveniente -a fin de reducir ruidos- disminuir exigencias formales orientadas (a falta de mejores herramientas) a asegurar la calidad, como serían, por ejemplo, el excesivo énfasis en la comprobación de los recursos propios (versus la disponibilidad de recursos y el impacto de éstos en el aprendizaje) o en la prohibición de fines de lucro (versus la transparencia y la responsabilidad en el cumplimiento de lo que se ofrece). 


\section{REFERENCIAS BiBLIOGRÁFICAS}

Núñez, Javier y Gutiérrez, Roberto, Classism, meritocracy and discrimination in the labor market: the case of Chile, Serie documentos de trabajo, abril 2004, Departamento de Economía, Universidad de Chile. [Sitio en Internet] Disponible en http://www.facea.uchile.cl/ FrameArea. $\operatorname{asp} ? \operatorname{cod}=78$

Teichler, Ulrich, "Educación Superior y Nuevo Desafío Socioeconómico en Europa", en: Burgen, Arnold, Goals and Purposes of Higher Education in the $21^{\text {st }}$ Century, Jessica Kingsley Publisher, Londres, 1996, Capítulo 9, traducido por Pedro Lafourcade. [Sitio en Intenet] Disponible en www.fceia.unr.edu.ar/labinfo/facultad/decanato/secretarias/ desarr_institucional/biblioteca_digital/biblioteca_univ_est_sociedad.htm, visitado en septiembre de 2004.

Lomas, Laurie, Does the development of mass education necessarily mean the end of quality?, Documento presentado en The Sixt QHE Seminar: The End of Quality?, Birmingham, 25-26 de mayo, 2001. [Documento en Internet] Disponible en www.qualityresearch-international.com/ papers/lomas.pdf

Harvey, Lee, Quality in Higher Education, Documento presentado en la Swedish Quality Conference, Göteborg, noviembre de 1999. [Documento en Internet] Disponible en www.shu.ac.uk/research/cre/ publications/goteborg.pdf

Lagrosen, Stefan, Seyyed-Hashemi y Leitner, Markus, "Examinations of the Dimensions of Quality in Higher Education", en: Quality Assurance in Education, Volumen 12, Número 2, 2004, pp. 61-69.

Randall, John, Aseguramiento de la Calidad: El Desafío de la Participación Masiva, Documento presentado en Seminario Internacional "Conocer y Situar al Estudiante. Reto a la Calidad", Santiago de Chile, 2002. [ Sitio en Internet] Disponible en www.cse.cl/ ASP/WEB_CSEpublic_pdf.asp

Raczynsky, Dagmar y Canales, Andrea, Demanda de Información sobre las Carreras de Pregrado en la Educación Superior Chilena, Documento 
presentado en Seminario Internacional "Información en la Educación Superior. Necesidades y Propuestas", Santiago de Chile, 2001. [Sitio en Internet] Disponible en www.cse.cl/ASP/WEB_CSEpublic_pdf.asp 